\title{
ALEXANDROFF'S MAPPING THEOREM FOR PARACOMPACT SPACES
}

By Keio NAGAMI

(Comm。by H. Tôyama)

This paper states Alexandroff's mapping theorem for paracompact spaces and gives a new characterization of paracompact spaces. A topological space $R$ is called to be approximated by complexes with geometric, natural or weak topology if, for every open covering $u$ of $k$, there exist respectively a simplicial complex $K$ with geometricl), natural ${ }^{2}$ or weak topology 3 and a continuous mapping $f \mid R \rightarrow K$ such that $\{f-1(s(p))\}$ refines $v$, where $S(p)$ denotes an open star with a centre $p$ and $p$ runs through all vertices of $K$. C. $H$. Dowker [ I] has proved that every paracompact Hausdorff space is approximated by geometric complexes or by natural ones. Our result (Theorem I) asserts that every paracompact Hausdorff space is approximated by complexes with weak topolo zy. Since weak topology is weaker than geometric and natural topology, ours includes Dowker's results.

Theorem 1. A paracompact Hausdorff space $R$ is approximated by complexes with weak topology.

Proof. Let $\pi$ be an arbitrary open covering of $R$, and then there exists a locally finite open covering $z=\left\{V_{\alpha}: \alpha \in A\right\}$ of $R$ which refines $v r$. Since $R$ is normal and then 30 is shrinkable, we can assume with no loss of generality that each $V_{\alpha}$ is an $F_{\sigma}$ -set. Therefore, there is a nonnegative real-valued continuous function $f_{\alpha}$ defined on $R$ such that $f_{\alpha}(x)>0$ if and only if $x \in V_{\alpha}$. We assoclate with each $V_{\alpha}$ a mark $p_{\alpha}$, and with $p_{\alpha}$ 's as vertices we construct the nerve $K$ of 30 such that $p_{\alpha}, \ldots$, $p_{\beta}$ are vertices of a simplex of $K$ if and only if the corresponding sets $V_{\alpha}, \ldots, V_{\beta}$ have a common point. We introduce into $K$ the weak topology. Let $x$ be an arbitrary point of $R$ and $A(x)$ be the set of indices such that $\mathbf{A}(\boldsymbol{x})=\left\{\alpha: \mathbf{x} \in V_{\alpha}\right\}$ 。 We construct a mapping $f \mid R \rightarrow K$ as follows:

$$
\begin{aligned}
f(x)= & \text { the centre of gravity of the } \\
& \text { vertices of }\left\{p_{\alpha}: \alpha \in A(x)\right\} \\
& \text { with the weights } f_{\alpha}(x) .
\end{aligned}
$$

Then $f$ is continuous: Let $W$ be an open neighborhood of $x$ such that $B(x)$ $=\left\{\alpha: W \cap V_{\alpha} \neq \phi\right\}$ is a finite set of indices. Let $K_{1}$, a subcomplex of $K$, be the nerve of $\left\{W_{\cap} \cap V_{\alpha}: \alpha \in B(x)\right\}$. Then evidently $f(W) \subset K_{1}$. Being $K_{1}$ a finite complex and $\boldsymbol{f}_{\alpha}$ continuous, it can easily be seen that $f \mid W \rightarrow K_{1}$ is continuous and hence $f \mid R \rightarrow K$ is continuous. From construction of $\mathrm{K}$, $s\left(p_{\alpha}\right)$ is nothing but the set of all points with a non-zero weight on $p_{\alpha}$, and hence $f$ is a baricentric 30 mapping, 1 . $e$.

$$
f^{-1}\left(S\left(p_{\alpha}\right)\right)=V_{\alpha} \quad \text { for all } \alpha \in A_{0}
$$

Thus $\left\{f^{-1}\left(s\left(p_{\alpha}\right)\right): \alpha \in A\right\}$ refines $\eta$. Q. E. D.

It is to be noted that a complex $K$ in the above can be reconstructed in more restricted type as follows.

Theorem 2. Each star $S(p)$ of $X$ can be of finite dimension.

Proof. Since a paracompact Hausdorff space is strongly screenable [5], we can assume with no loss of generality that zo stated in the above proof can be decomposed into a sequence $z_{i}, 1=1$, $2, \ldots$, such that:

$$
\begin{aligned}
& z=\bigcup_{i=1}^{\infty}, z_{i} \\
& z_{i}=\left\{v_{\alpha_{i}} ; \alpha_{i} \in A_{i}\right\}, \\
& v_{\alpha_{i}} \text { 's are, for fixed } i \text {, mutually } \\
& \text { disjoint. }
\end{aligned}
$$

Setting 


$$
v_{i}=\underbrace{}_{\alpha_{i} \in A_{i}} v_{\alpha_{i}},
$$

we get a countable open covering

$\left\{V_{i} ; i=1,2, \ldots\right\}$ of $R$. In general, a countable open covering of a countably paracompact 4 ) normal space has, as can easily be seen, a star-finite countable open covering which refines i.t. Therefore, $\left\{V_{i}\right\}$ can be refined by a star-finite open covering $\left\{W_{i}\right\}$ of $R_{\text {. }}$ With no loss of generality, as can easily be seen, we can assume that $V_{i} \supset W_{i}$ for every $i$. We construct an open covering zoof $R$ as follows:

$$
D_{0}=\bigcup_{i=1}^{\infty}\left\{w_{i} \cap V_{\alpha_{i}}: \alpha_{i} \in A\right\} \text {. }
$$

The nerve of $\not \supset$, say $K$, is what we sake for: It. is evident that $\overline{S(p)}$ is a subcomplex of $K$ whose combinatorial dimension is finite. In general, combinatorial dimension of a simplicial complex with weak topology coincides with its covering dimension [2]. Hence covering dimension of $S(p)$ is finite. Since the simplicial complex with weak topology is perfectly normal [4], the monotonicity of dimension holds in it. Therefore $S(p)$ is of finite dimension, $Q, E, D$.

Using Theorem 1, we get a characterization of paracompact spaces as well as Dowker's [1]。

Theorem 3. A Hausdorff space $R$ is paracompact if and only if $R$ is appro ximated by complexes with weak topology •

Proof. 'Only if' part has been shown in Theorem 1. Conversely, if $R$ is approximated by complexes with weak topology, there exist, for an arbitrary open covering zo of $R$, a simplicial complex $K$ with weak topology and a continuous mapping $f / R \rightarrow K$ such that $\left\{f^{-1}(s(p))\right\}$ refines $z p$. Since $\{S(p)\}$ is an open covering of $K$ and $x$ is paracompact [ 3 or 4 ], there exists a locally finite open covering

$\{U\}$ of $K$ which refines $\{S(p)\}$. Then $\left\{\mathrm{f}^{-1}(\mathrm{U})\right\}$ is a locally finite open covering of $R$ which refines $\left\{f^{-1}(S(p))\right\}$ and hence does zo. Q. E. D.
FOOTNOTE
1) See [2].
2) See $[1]$.
3) See $[6]$.

4) Following C. H. Dowker, a space is called countably paracompact if every countable open covering of it can be refined by a locally finite open covering.

\section{BIBLIOGRAPHY}

[1] C. H. Dowker: An extension of Alexandroff's mapping theorem, Bull. Amer. Math. Soc. 54(1948) 386-391.

[2] S. Lefschetz: Topics in Topology, Princeton, 1942.

[3] H. Miyazaki: Paracompactness of CW-complexes, Tohoku Math. J. 4 (1952) 309-313.

[4] K. Vorita: On spaces having the weak topology with respect to closed coverings, Proc. Japan Acad. 29(1954) 537-543.

[5] K. Nagami: Paracompactness and strong screenability, forthcoming in Nagoya Math. J.

[6] J. H. C. Whitehead: Combinatorial homotopy I, Bull. Amer. Math. Soc. 55(1949) 213-245.

Enime University, Katsuyama.

(*) Received November 20, 1954. 